\title{
Lagrangian Reduction on Homogeneous Spaces with Advected Parameters
}

Cornelia VIZMAN

Department of Mathematics, West University of Timişoara, Romania

E-mail:vizman@math.uvt.ro

Received August 14, 2014, in final form January 22, 2015; Published online January 29, 2015

http://dx.doi.org/10.3842/SIGMA.2015.009

\begin{abstract}
We study the Euler-Lagrange equations for a parameter dependent $G$-invariant Lagrangian on a homogeneous $G$-space. We consider the pullback of the parameter dependent Lagrangian to the Lie group $G$, emphasizing the special invariance properties of the associated Euler-Poincaré equations with advected parameters.
\end{abstract}

Key words: Lagrangian; homogeneous space; Euler-Poincaré equation

2010 Mathematics Subject Classification: 53D17; 53D20; 37K65; 58D05; 58D10

\section{Introduction}

The Euler-Poincaré (EP) equations arise via reduction of the variational principle for a right $G$-invariant Lagrangian $L: T G \rightarrow \mathbb{R}$. With a restricted class of variations, the extremals of the integral of the reduced Lagrangian $\ell: \mathfrak{g} \rightarrow \mathbb{R}$ correspond to extremals of the original variational problem for $L$ [9]. The EP equations are written for the right logarithmic derivative $\xi=\dot{g} g^{-1}=\delta^{r} g$ of the curve $g$ in $G$ as

$$
\frac{d}{d t} \frac{\delta \ell}{\delta \xi}+\operatorname{ad}_{\xi}^{*} \frac{\delta \ell}{\delta \xi}=0
$$

Here $\delta \ell / \delta \xi$ denotes the functional derivative of $\ell$, which depends on the choice of a space $\mathfrak{g}^{*}$ in duality with $\mathfrak{g}$, that is $\left(\frac{\delta \ell}{\delta \xi}, \eta\right)=\left.\frac{d}{d t}\right|_{t=0} \ell(\xi+t \eta)$ for all $\eta \in \mathfrak{g}$.

The case of a parameter dependent $G$-invariant Lagrangian $L: T G \times V^{*} \rightarrow \mathbb{R}$ is studied in [2]. The parameter space $V^{*}$ is a linear representation space of the Lie group $G$ and the associated EP equations include an advection equation for the parameter. These EP equations with advected parameters are applied to continuum theories in [6]. To integrate complex fluids in this setting, the case of an affine $G$-action on the parameter space $V^{*}$ is treated in [4]. The more general case when the parameter space is a smooth manifold $M$ acted on by $G$ is considered in [5], applied there to nematic particles. The reduced equations, called EP equations for symmetry breaking, written for the reduced Lagrangian $\ell: \mathfrak{g} \times M \rightarrow \mathbb{R}$, involve the cotangent momentum $\operatorname{map} J: T^{*} M \rightarrow \mathfrak{g}^{*}:$

$$
\frac{d}{d t} \frac{\delta \ell}{\delta \xi}+\operatorname{ad}_{\xi}^{*} \frac{\delta \ell}{\delta \xi}=J\left(\frac{\delta \ell}{\delta m}\right) .
$$

In this paper we generalize the Lagrangian reduction with avdected parameters from the Lie group setting to the homogeneous space setting. We use the approach from [10] that features the special invariance properties of the reduced equations written for the pullback Lagrangian to the Lie group $G$. Starting with a right $G$-invariant Lagrangian $\bar{L}: T(G / H) \rightarrow \mathbb{R}$, the reduced Lagrangian $\ell: \mathfrak{g} \rightarrow \mathbb{R}$ coming from its pullback to $T G$ must be invariant under the adjoint action of the Lie subgroup $H$ and under the addition action of its Lie algebra $\mathfrak{h}$. As a consequence, 
the EP equations (1.1) are now invariant under the following action of the group $C^{\infty}(I, H)$ on $C^{\infty}(I, \mathfrak{g})$ :

$$
h \cdot \xi=\operatorname{Ad}_{h} \xi+\delta^{r} h .
$$

The geodesic equations for invariant Riemannian metrics on Lie groups (Euler equations) correspond to reduced Lagrangians $\ell$ that are quadratic; a famous example is the ideal fluid flow as geodesic equations on the group of volume preserving diffeomorphisms [1] (more geodesic equations on diffeomorphism groups can be found for instance in [11]). The extension of Euler equations from Lie groups to homogeneous spaces is done in [8].

The plan of the paper is the following. In Section 2 we review a kind of logarithmic derivative for homogeneous spaces. Then we consider parameter dependent $G$-invariant Lagrangians on $G / H$. We treat the case of a linear action on the parameter space in Section 3, where a multidimensional Hunter-Saxton equation with advected parameter is obtained. We devote Section 4 to the EP equations for symmetry breaking, obtained for general actions on arbitrary parameter spaces. We obtain the affine EP equations as a special case. The examples are mainly on infinite dimensional homogeneous spaces, such as $\operatorname{Diff}\left(S^{1}\right) / S^{1}, \operatorname{Diff}(M) / \operatorname{Diff}_{\mathrm{vol}}(M)$, $\operatorname{Diff}(M) / \operatorname{Diff}_{\text {iso }}(M)$, and $C^{\infty}(M, G) / G$.

\section{Logarithmic derivative and EP equations}

The Euler-Lagrange (EL) equations associated to right invariant Lagrangians on Lie groups lead to the Euler-Poincaré (EP) equations involving reduced Lagrangians and written for right logarithmic derivatives of curves in the Lie group. In this section we recall how this works in homogeneous spaces of right cosets, following [10].

Given a smooth curve $\bar{g}: I \rightarrow G / H$, we compare the right logarithmic derivatives of two smooth lifts $g_{1}, g_{2}: I \rightarrow G$ of $\bar{g}$. Because there exists a smooth curve $h: I \rightarrow H$ such that $g_{2}=h g_{1}$, the logarithmic derivative $\delta^{r} g_{2}=\operatorname{Ad}_{h} \delta^{r} g_{1}+\delta^{r} h$ is obtained from $\delta^{r} g_{1}$ via the left action of the group $C^{\infty}(I, H)$ on $C^{\infty}(I, \mathfrak{g})$ :

$$
h \cdot \xi=\operatorname{Ad}_{h} \xi+\delta^{r} h .
$$

Hence the right logarithmic derivative for homogeneous spaces is multivalued:

$$
\bar{\delta}^{r}: C^{\infty}(I, G / H) \rightarrow C^{\infty}(I, \mathfrak{g}) / C^{\infty}(I, H), \quad \bar{\delta}^{r} \bar{g}=C^{\infty}(I, H) \cdot \delta^{r} g,
$$

where $g$ is any lift of $\bar{g}$.

The tangent bundle $T G$ of a Lie group $G$ carries a natural group multiplication. In the right trivialization $T G \cong \mathfrak{g} \times G$ the multiplication becomes $(\eta, h)(\xi, g)=\left(\operatorname{Ad}_{h} \xi+\eta, h g\right)$. Given a Lie subgroup $H$ of $G$, its tangent bundle $T H$ is a subgroup of $T G$. Let $\pi: G \rightarrow G / H$ denote the canonical projection. Then the surjective submersion $T \pi: T G \rightarrow T(G / H)$ is constant on right $T H$-cosets of $T G$ and descends to a canonical diffeomorphism between $T G / T H$ and $T(G / H)$.

The following are equivalent data: right $G$-invariant Lagrangian $\bar{L}$ on $T(G / H)$, left $T H$ invariant and right $G$-invariant Lagrangian $L$ on $T G$, as well as reduced Lagrangian $\ell$ on $\mathfrak{g}$ that is both $\mathfrak{h}$-invariant and $\operatorname{Ad}(H)$-invariant. The relation between the Lagrangians is $L=\bar{L} \circ T \pi$ and we call $L$ the pullback of $\bar{L}$.

Proposition 2.1 ([10]). If the reduced Lagrangian $\ell: \mathfrak{g} \rightarrow \mathbb{R}$ is $\mathfrak{h}$-invariant and $\operatorname{Ad}(H)$ invariant, i.e. $\ell\left(\operatorname{Ad}_{h} \xi+\eta\right)=\ell(\xi)$ for all $h \in H$ and $\eta \in \mathfrak{h}$, then the EP equations

$$
\frac{d}{d t} \frac{\delta \ell}{\delta \xi}+\operatorname{ad}_{\xi}^{*} \frac{\delta \ell}{\delta \xi}=0
$$

are $C^{\infty}(I, H)$-invariant for the action $(2.1)$. 
In other words, equation $(2.3)$ can be seen as an equation for $C^{\infty}(I, H)$-orbits in $C^{\infty}(I, \mathfrak{g})$, i.e. an equation for right logarithmic derivatives (2.2) of curves in the homogeneous space, so Proposition 2.1 can be reformulated as:

Proposition 2.2 ([10]). A solution of the EL equation for a right $G$-invariant Lagrangian $\bar{L}: T(G / H) \rightarrow \mathbb{R}$ is a curve in $G / H$ such that the logarithmic derivative of one of its lifts to $G$ satisfies the EP equation (2.3), with $\ell$ the reduced Lagrangian of the pullback $L: T G \rightarrow \mathbb{R}$.

This proposition admits a generalization to Lagrangians that are not necessarily right $G$ invariant. First we note the following property of a left $T H$-invariant Lagrangian $L: T G \rightarrow \mathbb{R}$ : if the curve $g$ in $G$ is a solution of the corresponding EL equation, then the curve $h g$ is also a solution of the EL equation, for any smooth curve $h$ in $H$. Indeed, each variation $g_{\varepsilon}$ of $g$ with fixed endpoints corresponds to a variation $(\varepsilon, t) \mapsto h(t) g_{\varepsilon}(t)$ of $h g$ with fixed endpoints.

Proposition 2.3. Let $L: T G \rightarrow \mathbb{R}$ be the pullback of the Lagrangian $\bar{L}: T(G / H) \rightarrow \mathbb{R}$ (i.e. $L$ is left TH-invariant). Then the following assertions hold:

(i) If the curve $g$ in $G$ is a solution of the EL equation for $L$, then it descends to the solution $\bar{g}=\pi \circ g$ of the EL equation for $\bar{L}$.

(ii) If the curve $\bar{g}$ in $G / H$ is a solution of the $E L$ equation for $\bar{L}$, then any lift $g$ of $\bar{g}$ is a solution of the EL equation for $L$.

Proof. Let $g$ be a solution of the EL equation for $L$ and $\bar{g}=\pi \circ g$. An arbitrary variation $\bar{g}_{\varepsilon}$ of $\bar{g}$ in $G / H$ with fixed endpoints can be lifted to a variation $g_{\varepsilon}$ of $g$ in $G$, but it doesn't necessarily have fixed endpoints. It only satisfies $g_{\varepsilon}(0) \in H g(0)$ and $g_{\varepsilon}(1) \in H g(1)$. We can achieve $g_{\varepsilon}(0)=g(0)$ by multiplying $g_{\varepsilon}(t)$ with $g(0) g_{\varepsilon}(0)^{-1}$ from the left. Moreover, we achieve $g_{\varepsilon}(1)=g(1)$ by multiplying the new variation $g_{\varepsilon}(t)$ with $g(1) g_{\varepsilon t}(1)^{-1}$ from the left. Now, using also the identity $L=\bar{L} \circ T \pi$, we get

$$
\left.\frac{d}{d \varepsilon}\right|_{0} \int \bar{L}\left(\bar{g}_{\varepsilon}(t), \dot{\bar{g}}_{\varepsilon}(t)\right) d t=\left.\frac{d}{d \varepsilon}\right|_{0} \int L\left(g_{\varepsilon}(t), \dot{g}_{\varepsilon}(t)\right) d t=0
$$

so that $\bar{g}$ is a solution of the EL equation for $\bar{L}$. This proves the first assertion.

The second assertion is straightforward, since a variation of $g$ in $G$ with fixed endpoints always descends to a variation in $G / H$ with fixed endpoints.

A special case is the geodesic equation for a right $G$-invariant Riemannian metric on $G / H$, i.e. Euler equation on homogeneous spaces [8]. The next examples are both of this type.

Example 2.4 ([7]). Let $(M, \mu)$ be a volume manifold. The homogeneous space of right cosets $\operatorname{Diff}(M) / \operatorname{Diff}_{\mathrm{vol}}(M)$ is the space of normalized volume forms. The right invariant metric on $\operatorname{Diff}(M) / \operatorname{Diff}_{\text {vol }}(M)$ induced by the degenerate $\dot{H}^{1}$ inner product on $\mathfrak{X}(M)$

$$
\langle u, v\rangle=\int_{M} \operatorname{div} u \operatorname{div} v \mu
$$

is isometric to the standard $L^{2}$ metric on an open subset of the sphere of radius $2 \sqrt{\operatorname{vol}(M)}$ in the Hilbert space $L^{2}(M)$. The isometry is

$$
\bar{\varphi} \in \operatorname{Diff}(M) / \operatorname{Diff}_{\mathrm{vol}}(M) \mapsto 2 \sqrt{\operatorname{Jac}(\varphi)} \in L^{2}(M),
$$

where the Jacobian of $\varphi \in \operatorname{Diff}(M)$ is computed w.r.t. $\mu$, i.e. $\varphi^{*} \mu=\operatorname{Jac}(\varphi) \mu$.

The geodesic equation is the multidimensional Hunter-Saxton equation

$$
\partial_{t} d(\operatorname{div} u)+d L_{u}(\operatorname{div} u)+(\operatorname{div} u) d(\operatorname{div} u)=0 .
$$


The reduced Lagrangian $\ell(u)=\frac{1}{2} \int_{M}(\operatorname{div} u)^{2} \mu$ on $\mathfrak{X}(M)$ has the required $\operatorname{Diff}_{\text {vol }}(M)$ - and $\mathfrak{X}_{\mathrm{vol}}(M)$-invariance properties. For $M=S^{1}$ one gets the Hunter-Saxton equation as geodesic equation on $\operatorname{Diff}\left(S^{1}\right) / S^{1}$.

The left action (2.1) involved in the definition of the right logarithmic derivative $\bar{\delta}^{r}$ on the homogeneous space $\operatorname{Diff}(M) / \operatorname{Diff}_{\mathrm{vol}}(M)$ is the action of the group $C^{\infty}\left(I, \operatorname{Diff}_{\mathrm{vol}}(M)\right)$ on $C^{\infty}(I, \mathfrak{X}(M))$ given by

$$
(\psi \cdot u)(t)=\left(\psi(t)^{-1}\right)^{*} u(t)+\delta^{r} \psi(t) \in \mathfrak{X}(M)
$$

since $\operatorname{Ad}_{\psi} u=\left(\psi^{-1}\right)^{*} u$. Here the right logarithmic derivative $\delta^{r} \psi=\partial_{t} \psi \circ \psi^{-1}$ is the time dependent (divergence free) vector field induced by the (volume preserving) isotopy $\psi(t)$. By Proposition 2.1 the Hunter-Saxton equation (2.4) is invariant under the action (2.5). This can be checked also by a direct computation.

Example 2.5 $([7])$. Let $(M, g)$ be a Riemannian manifold and $\operatorname{Diff}_{\text {iso }}(M)$ its group of isometries. The homogeneous space of right cosets $\operatorname{Diff}(M) / \operatorname{Diff}_{\text {iso }}(M)$ admits a right invariant metric induced by the degenerate inner product on $\mathfrak{X}(M)$

$$
\langle u, v\rangle=\int_{M}\left(L_{u} g, L_{v} g\right) \mu=\int_{M}\left(2\left(d u^{b}, d v^{b}\right)+4\left(\delta u^{b}, \delta v^{b}\right)-4 \operatorname{Ric}(u, v)\right) \mu .
$$

The reduced Lagrangian $\ell(u)=\frac{1}{2} \int_{M}\left|L_{u} g\right|^{2} \mu$ on $\mathfrak{X}(M)$ has the required $\operatorname{Diff}_{\text {vol }}(M)$ - and $\mathfrak{X}_{\mathrm{vol}}(M)$-invariance properties. The associated EP equation

$$
\begin{aligned}
4 d \delta u_{t}^{b} & +2 \delta d u_{t}^{b}-4 \operatorname{Ric}\left(u_{t}\right)+(\operatorname{div} u)\left(4 d \delta u^{b}+2 \delta d u^{b}-4 \operatorname{Ric}(u)\right) \\
& +L_{u}\left(4 d \delta u^{b}+2 \delta d u^{b}-4 \operatorname{Ric}(u)\right)=0
\end{aligned}
$$

is invariant under the action $(2.5)$ of $C^{\infty}\left(I\right.$, $\left.\operatorname{Diff}_{\text {iso }}(M)\right)$ on $C^{\infty}(I, \mathfrak{X}(M))$.

\section{$3 \quad$ EP equations with advected parameters}

Now we look at parameter dependent Lagrangians. First we treat the Lie group case, following [2], then we pass to homogeneous spaces.

\subsection{The case of Lie groups}

We consider a linear right action $\rho$ of the Lie group $G$ on the vector space $V$ and its dual left action $\rho^{*}$ on $V^{*}$. The corresponding Lie algebra actions on $V$ and $V^{*}$ are $\left.\frac{d}{d t}\right|_{0} \rho_{\exp (t \xi)}(v)=v \xi$ and $\left.\frac{d}{d t}\right|_{0} \rho_{\exp (t \xi)}^{*}(a)=\xi a$. If $\xi(t)=\delta^{r} g(t)$, then $a(t)=\rho_{g(t)}^{*}\left(a_{0}\right)$ is the unique solution of the differential equation with time-dependent coefficients $\dot{a}=\xi a, a(0)=a_{0}$. The diamond operation $\diamond: V \times V^{*} \rightarrow \mathfrak{g}^{*}$ is given by

$$
\langle v \diamond a, \xi\rangle:=\langle\xi a, v\rangle, \quad \text { for all } \quad \xi \in \mathfrak{g} .
$$

A right $G$-invariant Lagrangian $L: T G \times V^{*} \rightarrow \mathbb{R}$ (including the linear action on the parameter space in the second argument) has a reduced Lagrangian $\ell: \mathfrak{g} \times V^{*} \rightarrow \mathbb{R}$ so that

$$
\ell\left(v_{g} g^{-1}, \rho_{g}^{*}(a)\right)=L\left(v_{g}, a\right), \quad v_{g} \in T_{g} G .
$$

For fixed $a_{0} \in V^{*}$ the Lagrangian $L_{a_{0}}: T G \rightarrow \mathbb{R}$ is right invariant only under the isotropy subgroup $G_{a_{0}}$ of $a_{0} \in V^{*}$. 
Theorem 3.1 ([2]). The EL equations for $L_{a_{0}}$ on $G$ given by Hamilton's variational principle

$$
\delta \int_{t_{1}}^{t_{2}} L_{a_{0}}(g(t), \dot{g}(t)) d t=0
$$

can be expressed as EP equations on $\mathfrak{g} \times V^{*}$ with advected parameter:

$$
\frac{d}{d t} \frac{\delta \ell}{\delta \xi}+\operatorname{ad}_{\xi}^{*} \frac{\delta \ell}{\delta \xi}=\frac{\delta \ell}{\delta a} \diamond a, \quad \dot{a}=\xi a
$$

for the reduced Lagrangian $\ell$.

The main examples are the heavy top and the ideal compressible fluid. For the heavy top $G=\mathrm{SO}(3)$ and the parameter $\Gamma \in V^{*}=\mathbb{R}^{3}$ is the unit vector in the gravity direction in body representation. For the ideal compressible fluid $G=\operatorname{Diff}(M)$, with $M$ a Riemannian manifold, and the parameter $\rho \in V^{*}=C^{\infty}(M)^{*}$ is the fluid density in spatial representation. The reduced Lagrangians are $\ell(\Omega, \Gamma)=\frac{1}{2} \mathrm{I} \Omega \cdot \Omega-\Gamma \cdot \lambda$ for $\Omega \in \mathfrak{s o}(3)=\mathbb{R}^{3}$ in the first example, and $\ell(u, \rho)=\frac{1}{2} \int_{M}|u|^{2} \rho$ for $u \in \mathfrak{X}(M)$ in the second one.

\subsection{The case of homogeneous spaces}

Let $L: T G \times V^{*} \rightarrow \mathbb{R}$ be the pull-back of a $G$-invariant Lagrangian $\bar{L}: T(G / H) \times V^{*} \rightarrow \mathbb{R}$, hence $L$ is left $T H$-invariant and right $G$-invariant. If $\ell: \mathfrak{g} \times V^{*} \rightarrow \mathbb{R}$ is the reduced Lagrangian, then

$$
\ell(\xi, a)=L(\xi, a)=L\left(v_{h} \xi, a\right)=\ell\left(\operatorname{Ad}_{h} \xi+v_{h} h^{-1}, \rho_{h}^{*}(a)\right), \quad v_{h} \in T_{h} H .
$$

This proves the next proposition.

Proposition 3.2. The reduced Lagrangian $\ell: \mathfrak{g} \times V^{*} \rightarrow \mathbb{R}$ associated to the pullback of a parameter dependent right $G$-invariant Lagrangian on $G / H$ is $H$-and $\mathfrak{h}$-invariant:

$$
\ell\left(\operatorname{Ad}_{h} \xi+\eta, \rho_{h}^{*}(a)\right)=\ell(\xi, a), \quad h \in H, \quad \eta \in \mathfrak{h} .
$$

Lemma 3.3. The functional derivatives of the reduced Lagrangian $\ell: \mathfrak{g} \times V^{*} \rightarrow \mathbb{R}$ that has the invariance property (3.3) are equivariant:

$$
\frac{\delta \ell}{\delta \xi}\left(\operatorname{Ad}_{h} \xi+\eta, \rho_{h}^{*}(a)\right)=\operatorname{Ad}_{h^{-1}}^{*} \frac{\delta \ell}{\delta \xi}(\xi, a) \quad \text { and } \quad \frac{\delta \ell}{\delta a}\left(\operatorname{Ad}_{h} \xi+\eta, \rho_{h}^{*}(a)\right)=\rho_{h^{-1}} \frac{\delta \ell}{\delta a}(\xi, a) .
$$

Proof. We compute for $\zeta \in \mathfrak{g}$ :

$$
\begin{aligned}
\left(\frac{\delta \ell}{\delta \xi}\left(\operatorname{Ad}_{h} \xi+\eta, \rho_{h}^{*}(a)\right), \zeta\right) & =\left.\frac{d}{d t}\right|_{0} \ell\left(\operatorname{Ad}_{h} \xi+\eta+t \zeta, \rho_{h}^{*}(a)\right)=\left.\frac{d}{d t}\right|_{0} \ell\left(\xi+t \operatorname{Ad}_{h^{-1}}^{*}, a\right) \\
& =\left(\operatorname{Ad}_{h^{-1}}^{*} \frac{\delta \ell}{\delta \xi}(\xi, a), \zeta\right)
\end{aligned}
$$

Similarly we get that

$$
\begin{aligned}
\left(\frac{\delta \ell}{\delta a}\left(\operatorname{Ad}_{h} \xi+\eta, \rho_{h}^{*}(a)\right), b\right) & =\left.\frac{d}{d t}\right|_{0} \ell\left(\operatorname{Ad}_{h} \xi+\eta, \rho_{h}^{*}(a)+t b\right)=\left.\frac{d}{d t}\right|_{0} \ell\left(\xi, a+t \rho_{h^{-1}}^{*}(b)\right) \\
& =\left(\rho_{h^{-1}} \frac{\delta \ell}{\delta a}(\xi, a), b\right)
\end{aligned}
$$

for all $b \in V^{*}$. 
The path group $C^{\infty}(I, H)$ acts on $C^{\infty}\left(I, \mathfrak{g} \times V^{*}\right)$ by

$$
h \cdot(\xi, a)=\left(\operatorname{Ad}_{h} \xi+\delta^{r} h, \rho_{h}^{*}(a)\right) .
$$

This action has the property $h \cdot\left(\delta^{r} g, \rho_{g}^{*} a\right)=\left(\delta^{r}(h g), \rho_{h g}^{*} a\right)$ for any curve $g \in C^{\infty}(I, G)$.

Proposition 3.4. Given a reduced Lagrangian $\ell: \mathfrak{g} \times V^{*} \rightarrow \mathbb{R}$ that has the invariance property (3.3), the EP equation with advected parameters (3.2) is $C^{\infty}(I, H)$-invariant for the action (3.4).

Proof. We need the $G$-equivariance of the diamond operation:

$$
\operatorname{Ad}_{g}^{*}\left(v \diamond \rho_{g}^{*}(a)\right)=\rho_{g}(v) \diamond a
$$

that follows from $\rho_{g}^{*}(\xi a)=\left(\operatorname{Ad}_{g} \xi\right)\left(\rho_{g}^{*} a\right)$. Using also the following identities for $\alpha \in \mathfrak{g}^{*}$ :

$$
\operatorname{ad}_{\operatorname{Ad}_{h} \xi}^{*} \operatorname{Ad}_{h^{-1}}^{*} \alpha=\operatorname{Ad}_{h^{-1}}^{*} \operatorname{ad}_{\xi}^{*} \alpha, \quad \frac{d}{d t}\left(\operatorname{Ad}_{h^{-1}}^{*} \alpha\right)=-\operatorname{ad}_{\delta^{r} h}^{*} \operatorname{Ad}_{h^{-1}}^{*} \alpha,
$$

we compute

$$
\begin{aligned}
\left(\frac{d}{d t}\right. & \left.\frac{\delta \ell}{\delta \xi}+\operatorname{ad}_{\xi}^{*} \frac{\delta \ell}{\delta \xi}-\frac{\delta \ell}{\delta a} \diamond a\right)(h \cdot(\xi, a)) \\
& =\frac{d}{d t}\left(\operatorname{Ad}_{h^{-1}}^{*} \frac{\delta \ell}{\delta \xi}(\xi, a)\right)+\operatorname{ad}_{\operatorname{Ad}_{h} \xi+\delta^{r} h}^{*} \operatorname{Ad}_{h^{-1}}^{*} \frac{\delta \ell}{\delta \xi}(\xi, a)-\rho_{h^{-1}} \frac{\delta \ell}{\delta a}(\xi, a) \diamond \rho_{h}^{*} a \\
& =\operatorname{Ad}_{h^{-1}}^{*}\left(\frac{d}{d t} \frac{\delta \ell}{\delta \xi}+\operatorname{ad}_{\xi}^{*} \frac{\delta \ell}{\delta \xi}-\frac{\delta \ell}{\delta a} \diamond a\right)(\xi, a) .
\end{aligned}
$$

This ensures the $C^{\infty}(I, H)$-invariance of the EP equation with advected parameters.

Example 3.5. Let $M$ be a Riemannian manifold. As in Example 2.4 we focus on the group of volume preserving diffeomorphisms $\operatorname{Diff}_{\mathrm{vol}}(M)$ and the homogeneous space $\operatorname{Diff}(M) / \operatorname{Diff}_{\mathrm{vol}}(M)$ of volume forms with constant total volume.

We consider the parameter space $C^{\infty}(M)^{*}$, identified with $C^{\infty}(M)$ via the volume form $\mu$, hence the left $\operatorname{Diff}(M)$-action and its infinitesimal $\mathfrak{X}(M)$-action are

$$
\varphi \cdot \rho=\left(\rho \circ \varphi^{-1}\right) \operatorname{Jac}\left(\varphi^{-1}\right), \quad u \rho=-L_{u} \rho-\rho \operatorname{div} u=-\operatorname{div}(\rho u) .
$$

The diamond operation (3.1) becomes

$$
\diamond: C^{\infty}(M) \times C^{\infty}(M)^{*} \rightarrow \mathfrak{X}(M)^{*}, \quad f \diamond \rho=\rho d f \in \mathfrak{X}(M)^{*},
$$

where the dual of the space of vector fields is identified via the volume form $\mu$ with the space of differential 1-forms.

The reduced Lagrangian $\ell: \mathfrak{X}(M) \times C^{\infty}(M)^{*} \rightarrow \mathbb{R}$ given by

$$
\ell(u, \rho)=\frac{1}{2} \int_{M} \rho(\operatorname{div} u)^{2} \mu
$$

comes from a $\operatorname{Diff}(M)$-invariant parameter dependent Lagrangian on $\operatorname{Diff}(M) / \operatorname{Diff}_{\text {vol }}(M)$. Indeed, it satisfies the invariance property (3.3): for all $\psi \in \operatorname{Diff}_{\mathrm{vol}}(M)$ and $w \in \mathfrak{X}_{\mathrm{vol}}(M)$ we compute

$$
\ell\left(\operatorname{Ad}_{\psi} u+w, \psi \cdot \rho\right)=\frac{1}{2} \int_{M}\left(\left(\psi^{-1}\right)^{*} \rho\right)\left(\operatorname{div}\left(\psi^{-1}\right)^{*} u\right)^{2} \mu=\frac{1}{2} \int_{M} \rho(\operatorname{div} u)^{2} \psi^{*} \mu=\ell(u, \rho),
$$


using at step 2 the identity $\operatorname{div}\left(\psi^{*} u\right)=\psi^{*} \operatorname{div} u$ that holds for any volume preserving diffeomorphism $\psi$.

The EP equation with advected parameters (3.2) becomes

$$
\partial_{t} d(\rho \operatorname{div} u)+d L_{u}(\rho \operatorname{div} u)+d\left(\rho(\operatorname{div} u)^{2}\right)=0, \quad \partial_{t} \rho+\operatorname{div}(\rho u)=0 .
$$

By Proposition 3.4 this equation is $C^{\infty}\left(I, \operatorname{Diff}_{\mathrm{vol}}(M)\right)$-invariant for the joint action (3.4), namely

$$
\psi \cdot(u, \rho)=\left(\left(\psi^{-1}\right)^{*} u+\delta^{r} \psi,\left(\psi^{-1}\right)^{*}(\rho) \operatorname{Jac}\left(\psi^{-1}\right)\right)
$$

for curves $\psi$ in $\operatorname{Diff}_{\text {vol }}(M), u$ in $\mathfrak{X}(M)$, and $\rho$ in $C^{\infty}(M)$.

Example 3.6. One can consider as well the group $\operatorname{Diff}_{\text {iso }}(M)$ of isometries of $M$ as a subgroup of $\operatorname{Diff}(M)$, like in Example 2.5. The reduced Lagrangian $\ell: \mathfrak{X}(M) \times C^{\infty}(M)^{*} \rightarrow \mathbb{R}$ would be given by $\ell(u, \rho)=\frac{1}{2} \int_{M} \rho\left|L_{u} g\right|^{2} \mu$, coming from a $\operatorname{Diff}(M)$-invariant parameter dependent Lagrangian on the homogeneous space $\operatorname{Diff}(M) / \operatorname{Diff}_{\text {iso }}(M)$.

\section{$4 \quad$ EP equations for symmetry breaking}

One can replace the linear action of $G$ on a parameter vector space $V^{*}$ with an arbitrary action of $G$ on a parameter manifold $M$. This generalization of the EP equations with advected parameters, called EP equations for symmetry breaking, are presented in [5]. In this section we adapt these results to the case of homogeneous spaces.

\subsection{The case of Lie groups}

Let a Lie group $G$ act on the smooth manifold $M$ from the left, and let $\xi_{M} \in \mathfrak{X}(M)$ denote the infinitesimal generator of $\xi \in \mathfrak{g}$. Given a curve $g$ in $G$ starting at the identity, the curve $m(t)=$ $g(t) \cdot m_{0}$ is the unique solution of the differential equation with time-dependent coefficients

$$
\dot{m}=\xi_{M}(m), \quad m(0)=m_{0},
$$

where $\xi(t)=\delta^{r} g(t)$.

The cotangent momentum map $J: T^{*} M \rightarrow \mathfrak{g}^{*}$, defined by $\left(J\left(\alpha_{m}\right), \xi\right)=\left(\alpha_{m}, \xi_{M}(m)\right)$ for all $\alpha_{m} \in T_{m}^{*} M$, is $G$-equivariant for the cotangent and coadjoint actions: $J\left(g \cdot \alpha_{m}\right)=\operatorname{Ad}_{g}^{*} J\left(\alpha_{m}\right)$.

Given a right $G$-invariant Lagrangian $L: T G \times M \rightarrow \mathbb{R}$, i.e.

$$
L\left(v_{g} h, h^{-1} \cdot m\right)=L\left(v_{g}, m\right), \quad h \in G,
$$

its reduced Lagrangian $\ell: \mathfrak{g} \times M \rightarrow \mathbb{R}$ satisfies $L\left(v_{g}, m\right)=\ell\left(v_{g} g^{-1}, g \cdot m\right)$. The functional derivative $\frac{\delta \ell}{\delta \xi}$ takes values in $\mathfrak{g}^{*}$, while $\frac{\delta \ell}{\delta m}$ is a $\mathfrak{g}$-dependent section of $T^{*} M$.

Theorem 4.1 ([5]). The EL equations for the Lagrangian $L_{m_{0}}: T G \rightarrow \mathbb{R}$ are the EP equations for symmetry breaking

$$
\frac{d}{d t} \frac{\delta \ell}{\delta \xi}+\operatorname{ad}_{\xi}^{*} \frac{\delta \ell}{\delta \xi}=J\left(\frac{\delta \ell}{\delta m}\right), \quad \dot{m}=\xi_{M}(m)
$$

for the reduced Lagrangian $\ell: \mathfrak{g} \times M \rightarrow \mathbb{R}$.

Example $4.2([5])$. For an EP description of nematic particles one considers the canonical action of $G=\mathrm{SO}(3)$ on $M=\mathbb{R} P^{2}$. The $\mathrm{SO}(3)$-invariant Lagrangian is

$$
L: T \mathrm{SO}(3) \times \mathbb{R} P^{2} \rightarrow \mathbb{R}, \quad L(g, \dot{g}, m)=\frac{1}{2} j|\dot{g}|^{2}-\frac{\lambda}{2}\left\langle m, g^{-1} k\right\rangle^{2},
$$

where $j$ and $\lambda$ are constants, and $k$ the external force field, with reduced Lagrangian $\ell: \mathfrak{s o}(3) \times$ $\mathbb{R} P^{2} \rightarrow \mathbb{R}$ given by $\ell(\xi, m)=\frac{1}{2} j|\xi|^{2}-\frac{\lambda}{2}\langle m, k\rangle^{2}$. 


\subsection{The case of homogeneous spaces}

Let $L: T G \times M \rightarrow \mathbb{R}$ be the pull-back of the right $G$-invariant Lagrangian $\bar{L}: T(G / H) \times M \rightarrow \mathbb{R}$, hence $L$ is left $T H$-invariant (in the first argument) and right $G$-invariant (in both arguments simultaneously). The associated reduced Lagrangian $\ell: \mathfrak{g} \times M \rightarrow \mathbb{R}$ is both $H$ - and $\mathfrak{h}$-invariant:

$$
\ell\left(\operatorname{Ad}_{h} \xi+\eta, h \cdot m\right)=\ell(\xi, m), \quad h \in H, \quad \eta \in \mathfrak{h} .
$$

Proposition 4.3. Given a reduced Lagrangian $\ell: \mathfrak{g} \times M \rightarrow \mathbb{R}$ that has the invariance property (4.2), the EP equation for symmetry breaking (4.1) is invariant under the $C^{\infty}(I, H)$-action on $C^{\infty}\left(I, \mathfrak{g} \times T^{*} M\right)$ :

$$
h \cdot(\xi, m)=\left(\operatorname{Ad}_{h} \xi+\delta^{r} h, h \cdot m\right) .
$$

Proof. The equivariance property of the functional derivative $\frac{\delta \ell}{\delta \xi}$ from Lemma 3.3 holds, but also the following equivariance property of $\frac{\delta \ell}{\delta m}$ :

$$
\frac{\delta \ell}{\delta m}(h \cdot(\xi, m))=h^{-1} \cdot \frac{\delta \ell}{\delta m}(\xi, m) .
$$

Indeed, for any curve $c$ in $M$ with $c(0)=h \cdot m$ and $c^{\prime}(0)=w$, we get:

$$
\begin{aligned}
\left(\frac{\delta \ell}{\delta m}(h \cdot(\xi, m)), w\right) & =\left.\frac{d}{d t}\right|_{0} \ell\left(\operatorname{Ad}_{h} \xi+\delta^{r} h, c(t)\right)=\left.\frac{d}{d t}\right|_{0} \ell\left(\xi, h^{-1} \cdot c(t)\right) \\
& =\left(\frac{\delta \ell}{\delta m}(\xi, m), h^{-1} \cdot w\right)=\left(h^{-1} \cdot \frac{\delta \ell}{\delta m}(\xi, m), w\right) .
\end{aligned}
$$

Using also the equivariance of the cotangent momentum map, we compute

$$
\begin{aligned}
& \left(\frac{d}{d t} \frac{\delta \ell}{\delta \xi}+\operatorname{ad}_{\xi}^{*} \frac{\delta \ell}{\delta \xi}-J\left(\frac{\delta \ell}{\delta m}\right)\right)(h \cdot(\xi, m))=\frac{d}{d t}\left(\operatorname{Ad}_{h^{-1}}^{*} \frac{\delta \ell}{\delta \xi}\right)+\operatorname{ad}_{\operatorname{Ad}_{h} \xi+\delta^{r} h} \operatorname{Ad}_{h^{-1}}^{*} \frac{\delta \ell}{\delta \xi} \\
& -J\left(h^{-1} \cdot \frac{\delta \ell}{\delta m}\right)=\operatorname{Ad}_{h^{-1}}^{*}\left(\frac{d}{d t} \frac{\delta \ell}{\delta \xi}+\operatorname{ad}_{\xi}^{*} \frac{\delta \ell}{\delta \xi}-J\left(\frac{\delta \ell}{\delta m}\right)\right) .
\end{aligned}
$$

This shows the required invariance of the equation (4.1).

Example 4.4. This is a variation of Example 4.2 for the subgroup $H=S^{1}$ of $G=\operatorname{SO}(3)$ consisting of all rotations with axis $k$. Instead of the reduced Lagrangian $\ell(\xi, m)=\frac{1}{2} j|\xi|^{2}-$ $\frac{\lambda}{2}(m \cdot k)^{2}$ from Example 4.2 one can take $\ell(\xi, m)=\frac{1}{2} j\left|p_{k^{\perp}}(\xi)\right|^{2}-\frac{\lambda}{2}\langle m, k\rangle^{2}$, where $p_{k^{\perp}}$ denotes the orthogonal Euclidean projection onto the vectorial plane $k^{\perp} \subset \mathbb{R}^{3}$. It has the required invariance properties because $p_{k^{\perp}}(\eta)=0$ for all $\eta \in \mathfrak{h}$ (because $\eta$ is proportional to $k$ ) and $p_{k^{\perp}}\left(\operatorname{Ad}_{h} \xi\right)=\operatorname{Ad}_{h}\left(p_{k^{\perp}}(\xi)\right)$ for all $h \in H$. Indeed, for every rotation $h$ with axis $k$ and every $\eta \in \mathfrak{h}$,

$$
\ell\left(\operatorname{Ad}_{h} \xi+\eta, h \cdot m\right)=\frac{1}{2} j\left|p_{k^{\perp}}\left(\operatorname{Ad}_{h} \xi\right)\right|^{2}-\frac{\lambda}{2}\langle h \cdot m, k\rangle^{2}=\frac{1}{2} j\left|p_{k^{\perp}}(\xi)\right|^{2}-\frac{\lambda}{2}\langle m, k\rangle^{2}=\ell(\xi, m) .
$$

\subsection{Affine EP equations}

Now we consider the special case of an affine left $G$-action on a parameter space $V^{*}$ :

$$
\theta_{g}(a)=\rho_{g}^{*}(a)+c(g)
$$

where $c: G \rightarrow V^{*}$ is a group 1-cocycle for the action $\rho^{*}$, i.e.

$$
c(g h)=c(g)+\rho_{g}^{*} c(h) .
$$

Let $d c: \mathfrak{g} \rightarrow V^{*}$ be the associated Lie algebra 1-cocycle. If $\xi(t)=\delta^{r} g(t)$, then $a(t)=\theta_{g(t)}\left(a_{0}\right)$ is the unique solution of the differential equation with time-dependent coefficients $\dot{a}=\xi a+d c(\xi)$, $a(0)=a_{0}$. 
Remark 4.5. Let $d c^{\top}: V \rightarrow \mathfrak{g}^{*}$ be defined by $\left\langle d c^{\top}(v), \xi\right\rangle=\langle d c(\xi), v\rangle$. Then the cotangent momentum map for the affine action (4.3) of $G$ on $V^{*}$ can be written as

$$
J: T^{*} V^{*}=V^{*} \times V \rightarrow \mathfrak{g}^{*}, \quad J(a, v)=v \diamond a+d c^{\top}(v),
$$

because for all $\xi \in \mathfrak{g}$,

$$
(J(a, v), \xi)=\left(v, \xi_{V^{*}}(a)\right)=(v, \xi a+d c(\xi))=\left(v \diamond a+d c^{\top}(v), \xi\right) .
$$

The following result for a right $G$-invariant Lagrangian $L: T G \times V^{*} \rightarrow \mathbb{R}$ with reduced Lagrangian $\ell: \mathfrak{g} \times V^{*} \rightarrow \mathbb{R}$ is a special case of Theorem 4.1 and a generalization of Theorem 3.1.

Theorem 4.6 ([4]). The EL equations for $L_{a_{0}}: T G \rightarrow \mathbb{R}$ can be expressed as affine EP equations for the reduced Lagrangian $\ell$ :

$$
\frac{d}{d t} \frac{\delta \ell}{\delta \xi}+\operatorname{ad}_{\xi}^{*} \frac{\delta \ell}{\delta \xi}=\frac{\delta \ell}{\delta a} \diamond a+(d c)^{\top}\left(\frac{\delta \ell}{\delta a}\right), \quad \dot{a}=\xi a+d c(\xi)
$$

Spin systems. In [4] is shown that the affine EP equations for the action of the gauge group $C^{\infty}(M, G)$ on the space $V^{*}=\Omega^{1}(M, \mathfrak{g})$ of principal connections on the trivial bundle $M \times G$

$$
\theta_{g}(\gamma)=\operatorname{Ad}_{g} \gamma-d g g^{-1}
$$

can be used in the description of spin systems. The 1-cocycle is in this case the right logarithmic derivative

$$
c: C^{\infty}(M, G) \rightarrow \Omega^{1}(M, \mathfrak{g}), \quad c(g)=-d g g^{-1},
$$

so $d c(\xi)=-d \xi$ for all $\xi \in C^{\infty}(M, \mathfrak{g})$. The infinitesimal action involves the covariant derivative $d^{\gamma} \xi=d \xi+[\gamma, \xi]$, namely $\xi_{V^{*}}(\gamma)=-d^{\gamma} \xi$.

We fix a volume form on $M$, so $C^{\infty}\left(M, \mathfrak{g}^{*}\right)$ is a dual space to the gauge Lie algebra $C^{\infty}(M, \mathfrak{g})$, while $\mathfrak{X}\left(M, \mathfrak{g}^{*}\right)$ is a dual space to the parameter space $\Omega^{1}(M, \mathfrak{g})$. The cotangent momentum map (4.4) becomes $J(\gamma, \alpha)=-\operatorname{ad}_{\gamma}^{*} \alpha-\operatorname{div} \alpha=\operatorname{div}^{\gamma} \alpha$, since $d c^{\top}(\alpha)=\operatorname{div} \alpha$ and that the diamond map is $\alpha \diamond \gamma=-\operatorname{ad}_{\gamma}^{*} \alpha$. We can write now the affine EP equation on $C^{\infty}(M, \mathfrak{g}) \times$ $\Omega^{1}(M, \mathfrak{g})$ as

$$
\frac{\partial}{\partial t} \frac{\delta \ell}{\delta \xi}+\operatorname{ad}_{\xi}^{*} \frac{\delta \ell}{\delta \xi}=-\operatorname{div}^{\gamma} \frac{\delta \ell}{\delta \gamma}, \quad \dot{\gamma}+d^{\gamma} \xi=0 .
$$

For $M=\mathbb{R}^{3}$ and $G=\mathrm{SO}(3)$ one gets a macroscopic description of spin glasses [4]. For $M$ a real interval and $G=\operatorname{SE}(3)$, the Euclidean group of rigid motions, one gets an affine EP formulation of Kirchhoff's theory of rods (the Cosserat rod) in the case of potential forces [3].

Homogeneous spaces. Let $L: T G \times V^{*} \rightarrow \mathbb{R}$ be now the pull-back of a $G$-invariant Lagrangian $\bar{L}: T(G / H) \times V^{*} \rightarrow \mathbb{R}$. Because $L$ is left $T H$-invariant and right $G$-invariant, its reduced Lagrangian $\ell: \mathfrak{g} \times V^{*} \rightarrow \mathbb{R}$ is both $H$-and $\mathfrak{h}$-invariant:

$$
\ell\left(\operatorname{Ad}_{h} \xi+\eta, \theta_{h}(a)\right)=\ell(\xi, a), \quad h \in H, \quad \eta \in \mathfrak{h} .
$$

Proposition 4.7. Given a reduced Lagrangian $\ell: \mathfrak{g} \times V^{*} \rightarrow \mathbb{R}$ that has the invariance property (4.8), the affine EP equation (4.5) is invariant under the action of the path group $C^{\infty}(I, H)$ on $C^{\infty}\left(I, \mathfrak{g} \times V^{*}\right)$ by

$$
h \cdot(\xi, a)=\left(\operatorname{Ad}_{h} \xi+\delta^{r} h, \theta_{h}(a)\right) .
$$


Proof. It is a consequence of Proposition 4.3, but it can be shown also directly, as in the proof of Proposition 3.4, using the expression of the failure of $d c$ to be $G$-equivariant: $d c\left(\operatorname{Ad}_{g} \xi\right)-$ $\rho_{g}^{*} d c(\xi)=c(g) \operatorname{Ad}_{g} \xi$.

Example 4.8 (spin systems). Let $G$ be a Lie group and $\kappa$ an invariant inner product on its Lie algebra $\mathfrak{g}$. The reduced Lagrangians $\ell: C^{\infty}(M, \mathfrak{g}) \times \Omega^{1}(M, \mathfrak{g}) \rightarrow \mathbb{R}$ that depend only on the differential of the function $\xi \in C^{\infty}(M, \mathfrak{g})$ :

$$
\ell_{1}(\xi, \gamma)=\frac{1}{2} \int|[d \xi, \gamma]|^{2} \mu, \quad \ell_{2}(\xi, \gamma)=\frac{1}{2} \int|\kappa(d \xi, \gamma)|^{2} \mu, \quad \ell_{3}(\xi, \gamma)=\int\left(|d \xi|^{2}-|\gamma|^{2}\right) \mu
$$

all come from a Lagrangian on the homogeneous space $C^{\infty}(M, G) / G$ because all of them satisfy the invariance property (4.8). We check it for the middle Lagrangian for all $\xi \in C^{\infty}(M, \mathfrak{g})$, $h \in G$ and $\eta \in \mathfrak{g}$ (so $d h h^{-1}=0$ and $d \eta=0$ ):

$$
\ell_{2}\left(\operatorname{Ad}_{h} \xi+\eta, \theta_{h}(\gamma)\right) \stackrel{(4.6)}{=} \frac{1}{2} \int\left|\kappa\left(\operatorname{Ad}_{h} d \xi+d \eta, \operatorname{Ad}_{h} \gamma-d h h^{-1}\right)\right|^{2} \mu=|\kappa(d \xi, \gamma)|^{2} \mu=\ell_{2}(\xi, \gamma) .
$$

It follows that the corresponding EP equations (4.7) for spin systems are invariant under the action (4.9), hence (4.7) can be seen as an equation on $C^{\infty}(M, G) / G$.

This setting of affine EP reduction is used in [12] for the dynamical description of spacetime strands on homogeneous spaces. Covariant EP equations on homogeneous spaces provide another frame to describe the dynamics of space-time strands on homogeneous spaces.

\section{Acknowledgements}

The author is grateful to the referee for very helpful suggestions. This work was supported by a grant of the Romanian National Authority for Scientific Research, CNCS UEFISCDI, project number PN-II-ID-PCE-2011-3-0921.

\section{References}

[1] Arnold V., Sur la géométrie différentielle des groupes de Lie de dimension infinie et ses applications à l'hydrodynamique des fluides parfaits, Ann. Inst. Fourier (Grenoble) 16 (1966), 319-361.

[2] Cendra H., Holm D.D., Marsden J.E., Ratiu T.S., Lagrangian reduction, the Euler-Poincaré equations, and semidirect products, in Geometry of Differential Equations, Amer. Math. Soc. Transl., Vol. 186, Amer. Math. Soc., Providence, RI, 1998, 1-25, chao-dyn/9906004.

[3] Gay-Balmaz F., Holm D.D., Ratiu T.S., Variational principles for spin systems and the Kirchhoff rod, J. Geom. Mech. 1 (2009), 417-444, arXiv:0904.1428.

[4] Gay-Balmaz F., Ratiu T.S., The geometric structure of complex fluids, Adv. in Appl. Math. 42 (2009), 176-275, arXiv:0903.4294.

[5] Gay-Balmaz F., Tronci C., Reduction theory for symmetry breaking with applications to nematic systems, Phys. D 239 (2010), 1929-1947, arXiv:0909.2165.

[6] Holm D.D., Marsden J.E., Ratiu T.S., The Euler-Poincaré equations and semidirect products with applications to continuum theories, Adv. Math. 137 (1998), 1-81, chao-dyn/9801015.

[7] Khesin B., Lenells J., Misiołek G., Preston S.C., Geometry of diffeomorphism groups, complete integrability and geometric statistics, Geom. Funct. Anal. 23 (2013), 334-366, arXiv:1105.0643.

[8] Khesin B., Misiołek G., Euler equations on homogeneous spaces and Virasoro orbits, Adv. Math. 176 (2003), 116-144, math.SG/0210397.

[9] Marsden J.E., Ratiu T.S., Introduction to mechanics and symmetry. A basic exposition of classical mechanical systems, Texts in Applied Mathematics, Vol. 17, 2nd ed., Springer-Verlag, New York, 1999.

[10] Tiğlay F., Vizman C., Generalized Euler-Poincaré equations on Lie groups and homogeneous spaces, orbit invariants and applications, Lett. Math. Phys. 97 (2011), 45-60, arXiv:1008.4377.

[11] Vizman C., Geodesic equations on diffeomorphism groups, SIGMA 4 (2008), 030, 22 pages, arXiv:0803.1678.

[12] Vizman C., Invariant variational problems on homogeneous spaces, J. Geom. Phys., to appear. 